\title{
On an Application of a Modification of the Zincenko Method to the Approximation of Implicit Functions
}

IOANNIS K. ARGYROS

We use the Zincenko iteration to approximate implicit functions in a Banach space by solving a linear algebraic system of finite order. The non-linear equations involved contain a non-differentiable term. Our hypotheses are more general than Zabrejko and $\mathrm{Nguen}^{\prime}$ $[10]$, in this case.

Key words: Implicit function, Banach space

AMS subject classification: $47 \mathrm{D} 15,47 \mathrm{H17}, 65 \mathrm{~J} \mathrm{15,65 \textrm {BOS }}$

\section{Introduction}

Let $E, \wedge$ be Banach spaces and denote by $U\left(x_{0}, R\right)$ the closed ball with center $x_{0} \in E$ and of radius $R$ in $E$. We will use the same symbol for the norm $\|\cdot\|$ in both spaces. Let $P$ be a linear projection operator $\left(P^{2}=P\right)$ which projects $E$ on its subspace $E_{P}$ and set $Q=I-P$. Suppose that the non-linear operators $F(x, \lambda)$ and $G(x, \lambda)$ with values in $E$ are defined for $x \in D$, where $D$ is some convex subset of $E$ containing $U\left(x_{0}, R\right)$ and $\lambda \in U\left(\lambda_{0}, S\right)$. For each fixed $\lambda \in U\left(\lambda_{0}, S\right)$ the operator $\operatorname{PF}(z, \lambda)$ will be assumed to be Frechet differentiable for all $z \in D$. Then $P F(x, \lambda)$ will denote the Fréchet derivative of the operator $P F(z, \lambda)$ with respect to the argument $z$ at $z=x$. Moreover, we assume that $\left(P F^{\prime}\left(x_{0}, \lambda_{0}\right)\right)^{-1}$ exists and

$$
\begin{aligned}
& \left\|\left(P F^{\prime}\left(x_{0}, \lambda_{0}\right)\right)^{\prime 1}\left(P F^{\prime}(x, \lambda)-P F^{\prime}(y, \lambda)\right)\right\| \leq k_{1}(r, s)\|x-y\|, \\
& \left\|\left(P F^{\prime}\left(x_{0}, \lambda_{0}\right)\right)^{-1}\left(P F^{\prime}\left(x_{0}, \lambda\right)-P F^{\prime}\left(x_{0}, \lambda_{0}\right)\right)\right\| \leq k_{2}(s)\left\|\lambda-\lambda_{0}\right\|, \\
& \|\left(P F^{\prime}\left(x_{0}, \lambda_{0}\right)\right)^{-1}\left[(Q F(x, \lambda)+G(x, \lambda))-(Q F(y, \lambda)+G(y, \lambda)]\left\|s k_{3}(r, s)\right\| x-y \|,\right.
\end{aligned}
$$

for all $x, y \in U\left(x_{0}, r\right) \subset U\left(x_{0}, R\right)$ and $\lambda \in U\left(\lambda_{0}, s\right) \subset U\left(\lambda_{0}, S\right)$. Here $k_{1}, k_{2}$ and $k_{3}$ are nondecreasing functions on the intervals $[0, R] \times[0, S],[0, R]$ and $[0, R] \times[0, S]$, respectively. We use a modification of the Zincenko iteration [11]

$$
x_{n+1}(\lambda)=x_{n}(\lambda)-\left(P F\left(x_{n}(\lambda), \lambda\right)\right)^{-1}\left(F\left(x_{n}(\lambda), \lambda\right)+G\left(x_{n}(\lambda), \lambda\right)\right)(n \geq 0)
$$

to approximate a solution $x^{*}=x^{*}(\lambda)$ of the equation

$$
F(x, \lambda)+G(x, \lambda)=0
$$

By $x_{0}$ we mean $x_{0}(\lambda)$. That is $x_{0}$ depends on the $\lambda$ used in (4). 
It can easily be shown by induction on $n$ that under the above hypotheses $F\left(x_{n}(\lambda), \lambda\right)$ $+G\left(x_{n}(\lambda), \lambda\right)$ belongs to the domain of $P F^{\prime}\left(x_{n}(\lambda), \lambda\right)^{-1}$ for all $n \geq 0$. Therefore, if the inverses exist (at it will be shown later in Theorem 1), then the iterates $x_{n}$ can be computed for all $n \geq 0$.

Our assumptions (1) - (3) generalize the ones made by Zabrejko and Nguen [10], Yamamoto [9] and (for $G=0$ ) Potra and Pták [6]. The iterates generated by the above authors cannot be easily computed in infinite dimensional spaces since the inverses of the linear operators involved $(P=I$, then) may be to difficult or impossible to find. It is easy to see, however, that the solution of equations (4) reduces to solving certain operator equations in the space $E_{p}$. If, moreover, $E_{P}$ is a finite-dimensional space of dimension $N$, we obtain a system of linear algebraic equations of at most order $N$. Furthermore, several authors have treated the case when $G=0, P \neq I$ provided that $k_{1}$ and $k_{2}$ are constants (or not) $[1,2,4-6]$.

We provide sufficient conditions for the convergence of iteration (4) to a locally unique solution $x^{*}(\lambda)$ of equation (5) as well as several error bounds on the distances $\| x_{n+1}(\lambda)$ $-x_{n}(\lambda) \|$ and $\left\|x_{n}(\lambda)-\lambda^{*}(\lambda)\right\|$.

We need to define the functions

$$
\begin{aligned}
& a_{s}=k(s)\left\|\left(P F^{\prime}\left(x_{0}, \lambda_{0}\right)\right)^{-1}\left(F\left(x_{0}, \lambda\right)+G\left(x_{0}, \lambda\right)\right)\right\|\left(s=0 \text { if } \lambda=\lambda_{0}\right), \\
& \omega_{s}(r)=\int_{0}^{r} k_{1}(t, s) d s, k_{4}(s)=\int_{0}^{s} k_{2}(t) d t, k(s)=\left(1-k_{4}(s)\right)^{-1}
\end{aligned}
$$

provided that

$$
\begin{aligned}
& k_{4}(s)<1, \varphi_{s}(r)=a_{s}+k(s) \int_{0}^{r} \omega_{s}(t) d t-r, \\
& \psi_{s}(r)=k(s) \int_{0}^{r} k_{3}(t, s) d t, \chi_{s}(r)=\varphi_{s}(r)+\psi_{s}(r)
\end{aligned}
$$

and the iteration $\left(y_{0}=x_{0}, n \geq 0\right)$

$$
y_{n+1}(\lambda)=y_{s}(\lambda)-\left(P F^{\prime}\left(x_{0}, \lambda_{0}\right)\right)^{-1}\left(F\left(y_{n}(\lambda), \lambda\right)+G\left(y_{n}(\lambda), \lambda\right)\right) .
$$

\section{Convergence results}

We can now formulate the following result.

Theorem 1: Suppose that the function $\chi_{s}=\chi_{s}(r)$ has a unique zero $\rho^{*}=\rho_{s}^{*}$ in $[0, R]$ and $\chi_{s}(R) \leq 0$. Then the following statements are true.

(a) Equation (5) has a unique solution $x^{*}=x^{*}(\lambda) \in U\left(x_{0}, R\right)$ with $x^{*}(\lambda) \in U\left(x_{0}, \rho^{*}\right)$.

(b) The estimates

$$
\left\|y_{n+1}(\lambda)-y_{n}(\lambda)\right\| \leq v_{n+1}-v_{n}
$$

and

$$
\left\|y_{n}(\lambda)-x^{*}(\lambda)\right\| \leq p^{*}-v_{n}
$$

are true where the scalar sequence $\left\{v_{n}\right\}_{n \geq 0}$ is monotonically increasing and convergent to $p$ with

$$
v_{n+1}=d_{s}\left(v_{n}\right)\left(n \geq 0, v_{0}=0\right) \text { and } d_{s}(r)=r+\chi_{s}(r)
$$


Proof: $I t$ is a simple calculus to show that the sequence $\left\{v_{n}\right\}$ is monotonically increasing and convergent to $p^{*}$ (see also, $[10: p .675]$ ). Using induction to $n$ we will show that the estimate (7) is true, from which (8) will follow immediately. From (6) for $n=0$, we get

$$
\left\|y_{1}(\lambda)-y_{0}(\lambda)\right\| \leq\left\|\left(P F^{\prime}\left(x_{0}, \lambda\right)\right)^{-1}\left(F\left(x_{0}, \lambda\right)+G\left(x_{0}, \lambda\right)\right)\right\| \leq a_{s}=d_{s}(0)=v_{1}-v_{0} .
$$

That is. the estimate (7) is true for $n=0$. Let us assume that (7) is true for $n<k$. Then by (6), (1), (3), [10: p. 674] and the induction hypothesis we get

$$
\begin{aligned}
& \left\|y_{n+1}(\lambda)-y_{k}(\lambda)\right\| \\
& \leq\left\|\left(y_{k}(\lambda)-y_{k-1}(\lambda)\right)-\left(P F^{\prime}\left(x_{0}, \lambda_{0}\right)\right)^{-1}\left(P F\left(y_{k}(\lambda), \lambda\right)-P F\left(y_{k-1}(\lambda), \lambda\right)\right)\right\| \\
& +\left\|\left(P F^{\prime}\left(x_{0}, \lambda_{0}\right)\right)^{-1}\left\{\left(Q F\left(y_{k}(\lambda), \lambda\right)+G\left(y_{k}(\lambda), \lambda\right)\right)-\left(Q F\left(y_{k-1}(\lambda), \lambda\right)+G\left(y_{k-1}(\lambda), \lambda\right)\right)\right\}\right\| \\
& \leq \int_{0}^{1}\left\|\left(P F^{\prime}\left(x_{0}, \lambda_{0}\right)\right)^{-1}\left\{P F^{\prime}\left((1-t) y_{k-1}(\lambda)+t y_{k}(\lambda)\right)-P F^{\prime}\left(x_{0}, \lambda_{0}\right)\right\}\right\|\left\|y_{k}(\lambda)-y_{k-1}(\lambda)\right\| d t \\
& +\left\|\left(P F^{\prime}\left(x_{0}, \lambda_{0}\right)\right)^{-1}\left\{\left(Q F\left(y_{k}(\lambda), \lambda\right)+G\left(y_{k}(\lambda), \lambda\right)\right)-\left(Q F\left(y_{k-1}(\lambda), \lambda\right)+G\left(y_{k-1}(\lambda), \lambda\right)\right)\right\}\right\| \\
& \leq \int_{0}^{1} \omega\left((1-t) v_{k-1}+t v_{k}\right)\left(v_{k}-v_{k-1}\right) d t+\int_{v_{k-1}}^{v_{k}} k_{3}(t, s) d t \\
& \leq k(s)\left[\int_{v_{k-1}}^{v_{k}} \omega_{s}(t) d t+\int_{v_{k-1}}^{v_{k}} k_{3}(t, s) d t\right]=d_{s}\left(v_{k}\right)-d_{s}\left(v_{k-1}\right)=v_{k+1}-v_{k} .
\end{aligned}
$$

That is, the estimate (7) is true for $n=k$. Hence, $\left\{y_{n}(\lambda)\right\}$ is a cauchy sequence in a Banach space and as such converges to some $x^{*}(\lambda) \in U\left(x_{0}, \rho^{*}\right) \subset U\left(x_{0}, R\right)$. By letting $n \rightarrow \infty$ in (6) we deduce that $x^{-}(\lambda)$ is a solution of equation (S).

We will now show that $x^{*}(\lambda)$ is the unique solution of equation (5) in $U\left(x_{0}, R\right)$, by considering the sequences given by $\left(n \geq 0 ; z_{0} \in U\left(x_{0}, R\right)\right.$ and $\left.w_{0}=R^{\prime}\right)$

$$
z_{n+1}(\lambda)=z_{n}(\lambda)-\left(P F^{\prime}\left(x_{0}, \lambda_{0}\right)\right)^{-1}\left(F\left(z_{n}(\lambda), \lambda\right)+G\left(z_{n}(\lambda), \lambda\right)\right),
$$

and

$$
w_{n+1}=d_{s}\left(w_{n}\right) \text {. }
$$

It is enough to show that

$$
\left\|y_{n}(\lambda)-z_{n}(\lambda)\right\| \leq w_{n}-v_{n}, n \geq 0 .
$$

It is a simple calculus to show that the scalar sequence given by (11) is monotonically convergent to $\rho^{\circ}$. Hence, if for $z_{0}$ we choose the second solution $y^{\circ}(\lambda) \in U\left(x_{0}, r\right)$ of equation (S), then, by (12), $\| x^{*}(\lambda)-y^{*}(\lambda)$ il $s w_{n}-v_{n}$. That is, $x^{\top}(\lambda)=y^{*}(\lambda)$.

For $n=0$, (12) becomes $\left\|y_{0}-x_{0}\right\| \leq R-0=R$. Hence, (12) is true for $n=0$. Let us assume that (12) holds for $n \leq k$. Then by (6), (10) as before we get

$$
\begin{aligned}
& \left\|y_{k+1}(\lambda)-z_{k+1}(\lambda)\right\| \\
& \leq \quad\left\|\left(z_{k}(\lambda)-y_{k}(\lambda)\right)-\left(P F^{\prime}\left(\lambda_{0}, \lambda_{0}\right)\right)^{-1}\left(P F\left(z_{k}(\lambda), \lambda\right)-P F\left(y_{k}(\lambda), \lambda\right)\right)\right\| \\
& \quad+\left\|\left(P F^{\prime}\left(x_{0}, \lambda_{0}\right)\right)^{-1}\left\{\left(O F\left(z_{k}(\lambda), \lambda\right)+G\left(z_{k}(\lambda), \lambda\right)\right)-\left(Q F\left(y_{k}(\lambda), \lambda\right)+G\left(y_{k}(\lambda), \lambda\right)\right)\right\}\right\| \\
& \leq \int_{0}^{1}\left\|\left(P F^{\prime}\left(x_{0}, \lambda_{0}\right)\right)^{-1}\left\{P F^{\prime}\left((1-t) y_{k}(\lambda)+t z_{k}(\lambda)\right)-P F^{\prime}\left(x_{0}, \lambda_{0}\right)\right\}\right\|\left\|z_{k}(\lambda)-y_{k}(\lambda)\right\| d t
\end{aligned}
$$




$$
\begin{aligned}
&+\int_{v_{k}}^{u_{k} k_{k}}(t, s) d t \\
& s \int_{0}^{1} w_{s}\left((1-t) v_{k}+t w_{k}\right)\left(w_{k}-v_{k}\right) d t+\int_{v_{k}}^{w w_{k}} k_{3}(t, s) d t \\
& s k(s)\left[\int_{v_{k}}^{w_{k}} \omega(s) d t+\int_{v_{k}}^{w_{k}} k_{3}(t, s) d t\right]=d_{s}\left(w_{k}\right)-d_{s}\left(v_{k}\right)=w_{k+1}-v_{k+1} .
\end{aligned}
$$

That completes the proof of the theorem

We can now formulate the main result.

Theorem 2: Suppose that the hypotheses of Theorem ! are satisfied. Then the following statements are true.

(a) The sequence $\left(p_{n}\right)$ given by

$$
p_{n+1}=p_{n}+u_{s}\left(p_{n}\right)\left(p_{0}=0\right) \text { with } u_{s}(r)=-\chi_{s}(r) / \varphi_{s}^{\prime}(r)
$$

is monotonically increasing and converges to $\rho^{*}$.

(b) The iterates generated by (4) are well defined for all $n \geq 0$ and remain in $U\left(x_{0}, \rho^{*}\right)$.

(c) Moreover, the estimates

$$
\left\|x_{n+1}(\lambda)-x_{n}(\lambda)\right\| \leq p_{n+1}-p_{n}(n \geq I)
$$

and

$$
\left\|x_{n+1}(\lambda)-x^{*}(\lambda)\right\| \leq \rho^{*}-\rho_{n}(n \geq 0)
$$

are true.

Proof: Part (a) can be shown exactly as in Proposition 3 in [10: p. 677]. We will only show (13) since (14) will follow then from it immediately. For $n=0$ we get $\left\|x_{1}(\lambda)-x_{0}(\lambda)\right\|$ $s a_{s}=\rho_{1}-\rho_{0}$. That is, (13) is true for $n=0$. Let us assume that (13) is true for $n<k$. By the induction hypothesis

$$
\left\|x_{k}(\lambda)-x_{0}\right\| \leq \sum_{j=1}^{k}\left\|x_{j}(\lambda)-x_{j-1}(\lambda)\right\| \leq \sum_{j=1}^{k}\left(\rho_{j}-\rho_{j-1}\right)=\rho_{k},
$$

The Banach lemma on invertible operators, (2) and the estimate

$$
\left\|\left(P F^{\prime}\left(x_{0}, \lambda_{0}\right)\right)^{-1}\left(P F^{\prime}\left(x_{k}(\lambda) \lambda\right)-P F^{\prime}\left(x_{0}, \lambda_{0}\right)\right)\right\| \leq k(s) \omega_{s}\left(\rho_{k}\right) \leq k(s) \omega_{s}\left(\rho^{*}\right)=\varphi_{s}^{*}\left(\rho^{*}\right)+1 \leq 1,
$$

it follows that $P F^{\prime}(x, \lambda)$ is invertible for all $(x, \lambda) \in U\left(x_{0}, R\right) \times U\left(\lambda_{0}, S\right)$ and

$$
\begin{aligned}
& \left\|\left(P F^{\prime}\left(x_{k}(\lambda), \lambda\right)\right)^{-1} P F^{\prime}\left(x_{0}, \lambda_{0}\right)\right\| \\
& \quad s\left\|\left\{I+\left(P F^{\prime}\left(x_{0}, \lambda\right)\right)^{-1}\left(P F^{\prime}(x, \lambda)-P F^{\prime}\left(x_{0}, \lambda_{0}\right)\right)\right\}^{-1}\right\|\left\|\left(P F^{\prime}\left(x_{0}, \lambda\right)\right)^{-1} P F^{\prime}\left(x_{0}, \lambda_{0}\right)\right\| \\
& \quad s-k(s) / \varphi_{s}^{\prime}\left(\rho_{k}\right) .
\end{aligned}
$$

Then by (4), (1) - (3), (15) and the induction Hypothesis we get

$$
\begin{aligned}
& \left\|x_{k+1}(\lambda)-x_{k}(\lambda)\right\| \\
& =\left\|\left(P F^{\prime}\left(x_{k}(\lambda), \lambda\right)\right)^{-1}\left(F\left(x_{k}(\lambda), \lambda\right)+G\left(x_{k}(\lambda), \lambda\right)\right)\right\| \\
& \quad \leq \|\left(P F^{\prime}\left(x_{k}(\lambda), \lambda\right)\right)^{-1}\left\{F\left(x_{k}(\lambda), \lambda\right)-F\left(x_{k-1}(\lambda), \lambda\right)\right.
\end{aligned}
$$




$$
\begin{aligned}
& \left.-P F^{\prime}\left(x_{k-1}(\lambda), \lambda\right)\left(x_{k}(\lambda)-x_{k-1}(\lambda)\right)+G\left(x_{k}(\lambda) \lambda\right)-G\left(x_{k-1}(\lambda), \lambda\right)\right\} \| \\
s & \left\|\left(P F^{\prime}\left(x_{k}(\lambda) \lambda\right)\right)^{-1} P F^{\prime}\left(x_{0}, \lambda_{0}\right)\right\|\left[\int_{0}^{1} \|\left(P F^{\prime}\left(x_{0}, \lambda_{0}\right)\right)^{-1}\right. \\
& \quad\left\{\left(P F^{\prime}(1-t) x_{k-1}(\lambda)+t x_{k}(\lambda)\right)-P F^{\prime}\left(x_{k-1}(\lambda)\right)\right\}\|\| x_{k}(\lambda)-x_{k-1}(\lambda) \| d t \\
& \left.+\left\|\left(P F^{\prime}\left(x_{0}, \lambda_{0}\right)\right)^{-1}\left\{\left(Q F\left(x_{k}(\lambda), \lambda\right)+G\left(x_{k}(\lambda), \lambda\right)\right) \cdot\left(Q F\left(x_{k-1}(\lambda), \lambda\right)+G\left(x_{k-1}(\lambda), \lambda\right)\right)\right\}\right\|\right] \\
& s-\frac{k(s)}{\varphi_{s}^{\prime}\left(\rho_{k}\right)} \int_{0}^{1}\left\{\omega_{s}\left((1-t) \rho_{k-1}+t \rho_{k}\right)-\omega_{s}\left(\rho_{k-1}\right)\right\}\left(\rho_{k}-\rho_{k-1}\right) d t-\frac{1}{\varphi_{s}^{\prime}\left(\rho_{k}\right)}\left(\psi_{s}\left(\rho_{k}\right)-\Psi_{s}\left(\rho_{k-1}\right)\right) \\
& s \frac{\varphi_{s}\left(\rho_{k}\right)-\varphi_{s}\left(\rho_{k-1}\right)-\varphi_{s}^{\prime}\left(\rho_{k-1}\right)\left(\rho_{k}-\rho_{k-1}\right)+\Psi_{s}\left(\rho_{k}\right)-\Psi_{s}\left(\rho_{k-1}\right)}{\varphi_{s}^{\prime}\left(\rho_{k}\right)} \\
= & \rho_{k+1}-\rho_{k} .
\end{aligned}
$$

Hence, (13) is true for $n=k$

We will now derive some a posteriori error bounds for iteration (4). Let

$$
\begin{aligned}
& r_{n, s}=r_{n}=\left\|x_{n}(\lambda)-x_{0}\right\|, q_{n, s}(r)=q_{n}(r)=k_{1}\left(r_{n}+r, s\right), \\
& f_{n, s}(r)=f_{n}(r)=k_{3}\left(r_{n}+r, s\right) \text { for } r \in\left[0, R-r_{n}\right]
\end{aligned}
$$

and set

$$
a_{n, s}=a_{n}=\left\|x_{n+1}(\lambda)-x_{n}(\lambda)\right\|, b_{n, s}=b_{n}=k(s)\left(1-k(s) \omega_{s}\left(r_{n}\right)\right)^{-1} .
$$

Without loss of generality we assume that $a_{n}>0$. Then exactly as in Theorem 2 in [9:p. 989] we can show

Theorem 3: Suppose that the hypotheses of Theorem 1 are satisfied. Then the following statements are true.

(a) The equation

$$
r=a_{n}+b_{n} \int_{0}^{r}\left((r-t) q_{n}(t)+f_{n}(t)\right) d t
$$

has a unique positive zero $\rho_{n, s}^{*}=\rho_{n}^{*}$ in the interval $\left[0, R-r_{n}\right], n \geq 0$ and $\rho_{0}^{*}=\rho^{*}$.

(b) The estimates

$$
\left\|x_{n}(\lambda)-x^{*}(\lambda)\right\| \leq \rho_{n}^{*} \leq \begin{cases}\left(\rho-\rho_{n}\right) a_{n} / \Delta \rho_{n} & \text { for } n \geq 0 \\ \left(\rho^{*}-p_{n}\right) a_{n-2} / \Delta p_{n-1} & \text { for } n \geq 1 \\ \rho^{*}-\rho_{n} & \text { for } n \geq 0\end{cases}
$$

are true, where $\Delta \rho_{n}=\rho_{n+1}-\rho_{n}$. That is, our bound (16) is sharper than Miel-type bounds $[3,7]$ and more general than the corresponding one in [9: p. 989] (for $P=I)$.

\section{REFERENCES}

[1] ARGYROS.I.K.: On Newton's method and nondiscrete mathematical induction. Bull. Austr. Math. Soc. 38 (1988). $131-140$.

[2] BALAzS, M., and G. GOLDNER : On the method of the cord and on a modification of it for the solution of nonlinear operator equations. Stud. Cerc. Mat. 20 (1968). $981-990$. 
[3] GRAGC, W. B., and R. A. TAPIA: Optimal error bounds for the Neuton-kiantoro. vich Theorem. SIAM J. Numer. Anal. 1(1974), $10-13$

(4) KANTOROVICH, L. V., and G. P. AKILOV: Functional Analysis in Normed Spaces. New York: Pergamon Press 1964.

[5] Krasnoselskil, M. A., Vainikko, G. M., Zabrejko, P. P., et al.: The apprximate solution of operator equations [Russian J. Mocow: Nauka 1969.

[6] POTRA, F. A., and V. PTAK: Sharp error bounds for Newton's process. Numer. Math. 34 (1980). $63-72$.

[7] RHEINBOLDT, W. C.: A unified convergence theory for a class of iterative processes. SIAM J. Numer. Anal. 5 (1968), 42 - 63.

[8] YaMAMOTO, T.: A method for finding sharp error bounds for Newton's method under the Kantorovich assumptions. Num. Math. 44 (1986), $203-220$.

[9] YAMМMOTO, T. A note on a posteriori error bound of Zabrejko and $N_{\text {guen for }}$ in cenko's iteration. Numer. Funct. Anal. and Optimiz. 9 (1987). 987 - 994.

[10] ZABREJKO.P.P., and D. F. NGUEN: The majorant method in the theory of NewtonKantorovich npproximations and the Ptak error estimates. Numer. Funct. Anal. and Optim. 9 (1987), 671-674.

[11] ZINCENKO, A. I.: Some approximate methods of solving equations with nondifferentiable operators (Ukrainian). Dopovidi Akad. Nauk Ukrain. RSR (1963), 156 - 161.

Received 02. 10. 1989; in revised form

Author's address:

Prof. Dr. Ioannis K. Argyros

Cameron University

Department of Mathematics

Lawton, OK 73505-6377, U.S.A. 